\title{
Measurement of the three- dimensional velocity distribution of Stark-decelerated Rydberg atoms
}

\section{Conference Paper}

\section{Author(s):}

Vliegen, E.; Limacher, P.; Merkt, Frédéric

Publication date:

2006

Permanent link:

https://doi.org/10.3929/ethz-b-000000830

\section{Rights / license:}

In Copyright - Non-Commercial Use Permitted

\section{Originally published in:}

The European Physical Journal D 40(1), https://doi.org/10.1140/epjd/e2006-00095-1 


\title{
Measurement of the three-dimensional velocity distribution of Stark-decelerated Rydberg atoms
}

\author{
E. Vliegen, P.A. Limacher, and F. Merkt ${ }^{\mathrm{a}}$ \\ Laboratorium für Physikalische Chemie, ETH Zurich, HCI Hönggerberg, 8093 Zurich, Switzerland
}

\author{
Received 1st December 2005 / Received in final form 14 February 2006 \\ Published online 28 April 2006 - (c) EDP Sciences, Società Italiana di Fisica, Springer-Verlag 2006
}

\begin{abstract}
The full three-dimensional velocity distributions of decelerated and accelerated particles in a Stark decelerator for Rydberg atoms and molecules have been measured. In the experiment, argon atoms in a supersonic beam are excited to low-field and high-field seeking Stark states with principal quantum number in the range $n=15$ to 25 and are decelerated in a $3 \mathrm{~mm}$ long decelerator consisting of four electrodes on which time-dependent voltages are applied. The time dependence of the resulting inhomogeneous electric field is chosen such that the decelerating force acting on the high-field seeking states is maximized at each point along the trajectories. The three-dimensional velocity distribution of the atoms before and after the deceleration is determined by measuring times of flight and two-dimensional images of the atomic cloud on the detector. Under optimal deceleration conditions, the decrease in kinetic energy in the longitudinal dimension amounts to $1.0 \times 10^{-21} \mathrm{~J}$ and the increase in mean kinetic energy in the transverse dimensions is only $1.0 \times 10^{-23} \mathrm{~J}$. The corresponding temperatures of $100 \mathrm{mK}$ and $300 \mathrm{mK}$ in the two transverse dimensions are sufficiently low that trapping can be envisaged. The possibility of focusing a Rydberg atom beam is demonstrated experimentally.
\end{abstract}

PACS. $32.60 .+\mathrm{i}$ Zeeman and Stark effects $-39.10 .+\mathrm{j}$ Atomic and molecular beam sources and techniques

\section{Introduction}

In recent efforts to create samples of translationally cold molecules, experiments using supersonic beams have played an important role [1]. In supersonic beams, the vibrational and electronic internal degrees of freedom have relaxed to the ground state, only a few rotational levels are populated, and the translational temperature is typically around $1 \mathrm{~K}$. However, the mean lab-frame velocity of the particles in the beam typically amounts to several hundreds of $\mathrm{m} / \mathrm{s}$. Electrostatic deceleration of polar particles in supersonic beams using long arrays of dipolar stages has proven to be a viable strategy to reduce the mean velocity to close to zero in the lab frame [2]. As a result, high resolution spectroscopy [3] and molecular optics experiments [4] could be performed on such samples.

In similar experiments on argon atoms in Rydberg states the kinetic energy in the direction of the beam propagation could be reduced by a comparable amount (i.e. by about $10^{-21}$ Joule) using a single deceleration stage of $3 \mathrm{~mm}$ length and moderate electric field strengths of less than $1000 \mathrm{~V} / \mathrm{cm} \mathrm{[5].} \mathrm{The} \mathrm{initial} \mathrm{velocity} \mathrm{of} \mathrm{the} \mathrm{atoms}$ in the beam $(590 \mathrm{~m} / \mathrm{s})$ could be reduced by about $5 \%$. Rydberg particles offer several advantages for deceleration experiments: (1) the electric dipole moments induced

\footnotetext{
a e-mail: frederic.merkt@ethz.ch
}

by external electric fields are large and scale as $n^{2}$ (at $n=20$, the field-induced dipole moment of a blue-shifted Stark state is $\sim 1000$ Debye); (2) the deceleration does not require the atoms or molecules to have a permanent dipole moment in the ground state; (3) all atoms and molecules have Rydberg states and thus the deceleration method is, at least in principle, universally applicable [5-8]. In this contribution, time-of-flight (TOF) measurements of the longitudinal velocity distribution of decelerated atoms and imaging measurements of the transverse velocity distribution are presented together with an analysis based on numerical simulations of the particle trajectories. From the measurements and the particle trajectory simulations, the full three-dimensional velocity distribution of the excited atoms can be reconstructed. A specific goal was to compare the forces acting on the Rydberg particles in the dimensions perpendicular to the beam propagation axis to those acting along the direction of the supersonic beam.

\section{Deceleration method}

A detailed discussion of the deceleration of Rydberg particles can be found in references [5-8] and is only described here briefly. The deceleration method is based on the fact that the energies of the members of the high- $\ell$ manifold of 
(a)

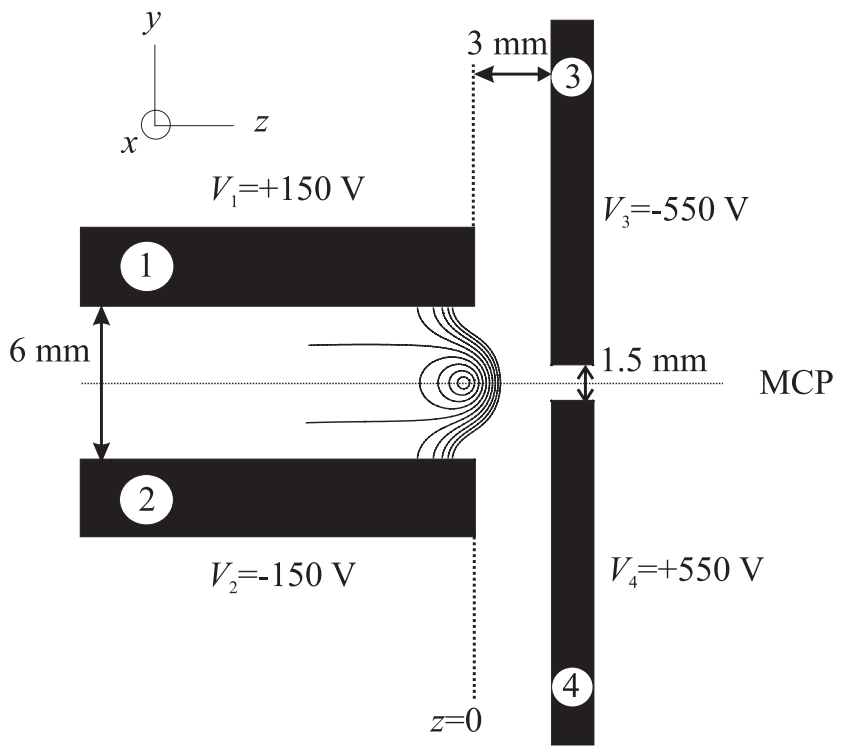

(b)

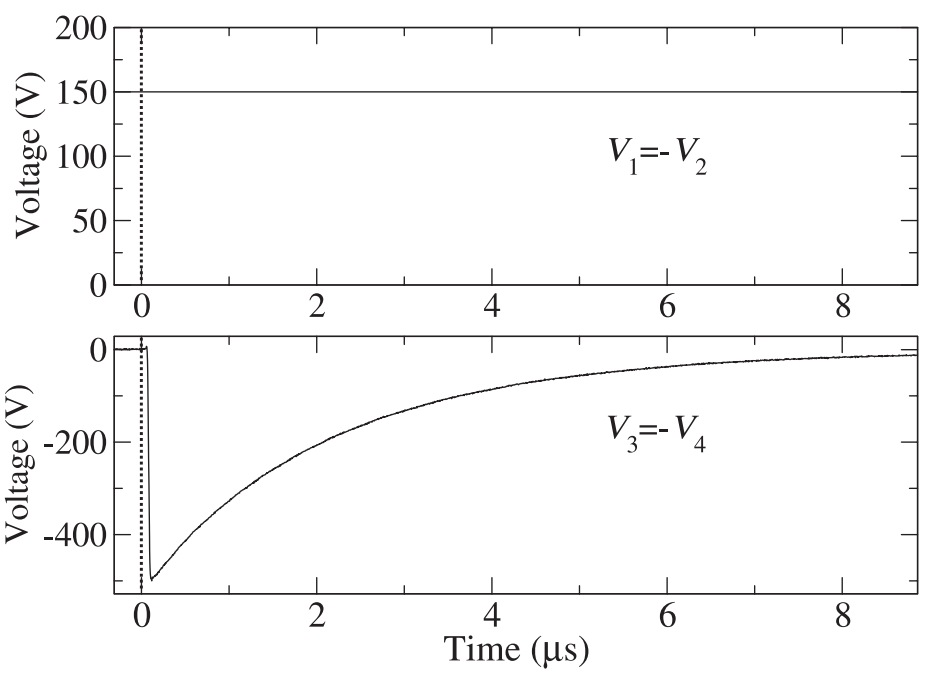

Fig. 1. (a) Schematic overview of the experimental set-up. The skimmed supersonic beam of the sample gas (argon) propagates in the $z$-direction halfway between the metallic electrodes. The VUV laser beam propagates in the $x$-direction parallel to the plate surfaces and perpendicular to the atomic beam. The photoexcitation point can be shifted by translating the electrode set-up in the $z$-direction. For the voltages indicated in the figure, the solid lines represent lines of constant electric field ranging from $100 \mathrm{~V} / \mathrm{cm}$ to $1000 \mathrm{~V} / \mathrm{cm}$ in steps of $100 \mathrm{~V} / \mathrm{cm}$. (b) Time dependence of the electrode potentials in the deceleration experiments in time-dependent electric fields. The excitation time is indicated at $t=0 \mu \mathrm{s}$ by the dotted line. The electric field vectors between the front plates and between the back plates point in opposite directions.

Rydberg states of atoms and molecules depend linearly on the strength $F$ of an externally applied electric field as a result of the Stark effect [9]. In an inhomogeneous electric field, the force $\mathbf{f}$ which acts on the Rydberg particles can be expressed in atomic units to a good approximation as:

$$
\mathbf{f}=-\frac{3}{2} n k \nabla F
$$

where $n$ is the principal quantum number, $k$ a quantum number which labels the Stark states and which runs from $-(n-1-|m|)$ to $(n-1-|m|)$ in steps of 2 , and $F$ is the electric field strength in atomic units (1 atomic unit of field $=5.142 \times 10^{9} \mathrm{~V} / \mathrm{cm}$ ). Integration of the force along the trajectories of the particles gives their change in kinetic energy. In nonhydrogenic systems, the maximum electric field at which equation (1) is still valid is the Inglis-Teller field $F_{\mathrm{IT}}$, i.e., the field given by $F_{\mathrm{IT}}=1 / 3 n^{5}$ at which the Stark manifolds of adjacent $n$ start to overlap energetically [9]. Above this field, the efficiency of the deceleration method is significantly reduced because of adiabatic passage of the avoided crossings between the Stark states [8].

Whether acceleration or deceleration is observed depends on the sign of the product $k \nabla F$ in equation (1). In the cases discussed here, the electric field strength $F(z)$ increases in the direction of propagation of the supersonic beam (see Fig. 1a) and $\mathrm{d} F / \mathrm{d} z>0$. Consequently, the Rydberg particles in states with $k>0$ (low-field seeking states) decelerate and those in states with $k<0$ (high- field seeking states) accelerate. States with $k=0$ have a field-independent energy and can be used as references to quantify the deceleration/acceleration behaviour.

Changes in the longitudinal velocity can be determined from a measurement of the times of flight of the particles. Because in our set-up the electric field is also inhomogeneous in one of the transverse directions, the velocity in this direction will change according to equation (1). Recording images of the beam after the deceleration represents a convenient way to characterize these changes.

\section{Experimental set-up and techniques}

In the experiments, argon atoms in a supersonic beam are excited from the ${ }^{1} \mathrm{~S}_{0}$ electronic ground state to Rydberg states of principal quantum number in the range $n=15$ to 25 located below the ${ }^{2} \mathrm{P}_{3 / 2}$ ionisation threshold in the presence of an electric field. After photoexcitation, the Rydberg atoms traverse a region of space in which an inhomogeneous electric field is applied. Depending on whether the Rydberg states are in a low- or a high-field seeking Stark state they are subject to a decelerating or an accelerating force. The effect of this force on the particle trajectories is then monitored by recording both the times that the atoms take to reach the detector and the images of the two-dimensional spatial distribution of the atoms on the detector. 
Single-photon excitation from the ground state of argon to Rydberg states necessitates vacuum-ultraviolet (VUV) radiation. In the experiment, a VUV laser is used that is broadly tunable $(8 \mathrm{eV}$ to $16 \mathrm{eV})$ and has a narrow bandwidth $\left(0.008 \mathrm{~cm}^{-1}\right)$ with an intensity corresponding to $10^{8}$ to $10^{9}$ photons per pulse [10] at a repetition rate of $25 \mathrm{~Hz}$. The three main components of our experimental set-up, the gas beam, the detection system and the electrodes, are described in the following subsections.

\subsection{The supersonic gas beam}

A supersonic beam is generated by expanding argon gas at a stagnation pressure of $\sim 2$ bar through a pulsed solenoid valve (General Valves, Series 9) into vacuum. The valve has a diameter of $0.5 \mathrm{~mm}$ and is open for approximately $250 \mu \mathrm{s}$. The beam passes through a $0.5 \mathrm{~mm}$ diameter skimmer located $12 \mathrm{~cm}$ downstream of the nozzle orifice. The skimmer orifice is small enough to maintain more than two orders of magnitude pressure difference between the nozzle chamber (background pressure $10^{-7}$ mbar, operating pressure $10^{-4}$ mbar) and the magnetically shielded photoexcitation region and flight tube (background pressure $5 \times 10^{-8}$ mbar, operating pressure $3 \times 10^{-7}$ mbar .

The skimmed beam intersects the VUV laser beam at right angles $\sim 7 \mathrm{~cm}$ away from the tip of the skimmer, and the excited atoms fly an additional $\sim 20 \mathrm{~cm}$ to the detector. The longitudinal velocity distribution is centered around $590 \mathrm{~m} / \mathrm{s}$ and has a width corresponding to a temperature of $\sim 0.6 \mathrm{~K}$ as inferred from the time-of-flight (TOF) measurements. The transverse velocity distribution is typically narrower and is determined by: (1) the collimation of the beam when it passes through the skimmer, (2) the spot size of the VUV laser beam, and (3) the interaction of the beam with the skimmer, as will be explained in Section 4.1.

\subsection{Detection system}

The detector is a $40 \mathrm{~mm}$ diameter microchannel plate (MCP) detector where the metal anode has been replaced by a phosphor screen. When an ion or an electron impinges on the MCP detector, secondary electrons are produced, which are accelerated toward the phosphor screen where they induce a fluorescence signal at the point of impact on the MCP detector. In our experiments, we detect the ionisation signal corresponding to the field ionisation of the Rydberg particles when they approach the detector surface. The fluorescence of the phosphor screen is monitored by a CCD camera $(640 \times 480$ pixels $)$ which has a resolution of 15 pixels $/ \mathrm{mm}$. The images give a faithful representation of the two-dimensional transverse spread of the atomic beam.

The detector has been constructed so that TOF spectra, corresponding to the integrated signal across the surface of the detector, can be measured simultaneously to the CCD-camera images at a time resolution of a few nanoseconds. In this way the transverse and longitudinal velocity distributions of incoming particles can be derived from a single measurement.

\subsection{Electrode set-up}

The electrode set-up is depicted in Figure 1a and has been described in detail in reference [5]. Each of the four electrodes can be set independently to any desired voltage up to $5000 \mathrm{~V}$. The deceleration can be improved by applying time-dependent voltages on the electrodes and a typical voltage sequence is displayed in Figure 1b. The resulting distributions of electric field strength along the $z$-axis at $70 \mathrm{~ns}$ and $2 \mu$ s after photoexcitation are shown in Figure $2 \mathrm{a}$. To optimize the deceleration, the potentials on the third and fourth electrodes are reduced continuously so that the Rydberg particles are always situated in the region of space indicated by the vertical lines in Figure 2a where the electric field is between zero and $F_{\mathrm{IT}}$. In this way, complications associated with the avoided crossings between Stark states at high fields are avoided [8].

To assess the changes in velocity in the direction perpendicular to the VUV beam, the $y$-dependence of the electric field strength in the $x y$-plane must be considered. The distributions along the $y$-axis are drawn in Figure $2 \mathrm{~b}$ for the longitudinal positions $z=-0.4 \mathrm{~mm}$ (solid line) and $z=0$ (dashed line). The photoexcitation region was located between these two points. In the direction parallel to the VUV laser beam (the $x$-axis in Fig. 1a), the electric field is homogeneous because the electrodes are much longer $(2 \mathrm{~cm})$ in this direction than the spread of the atomic cloud $(\sim 1 \mathrm{~mm})$. The particles therefore neither decelerate nor accelerate in this direction and the corresponding velocity and spatial distributions of the Rydberg atoms are independent of the $n$ and $k$ quantum numbers of the excited state. From the distribution of equi-field lines in Figure $1 \mathrm{a}$ and the field distribution in the $x y$-planes depicted in Figure 2b, one can see that the electrode setup acts as a concave cylindrical lens for high-field seeking states $(k<0)$ which are accelerated along the $z$-axis and as a convex lens for the low-field seeking states that are decelerated. This behaviour will be discussed in more detail in Section 4.1. With this single deceleration stage, a kinetic energy reduction of $60 \mathrm{~cm}^{-1}$ (in units of $h c$ ) can be achieved routinely in the range $n=15$ to 18 .

\subsection{Simulations}

To quantify the deceleration behaviour, particle trajectory simulations as described in [5] were carried out to determine the three-dimensional trajectories of the Rydberg particles. The force in the $x$-direction was taken to be zero in accord with the remarks made above. The TOF profiles and the images of the Rydberg atom beam on the detector can be extracted from the trajectories for direct comparison with the experimental results. Table 1 lists the parameters that were used in the simulations. These parameters were not adjusted but could either be measured directly or were known from earlier experiments. 
(a)

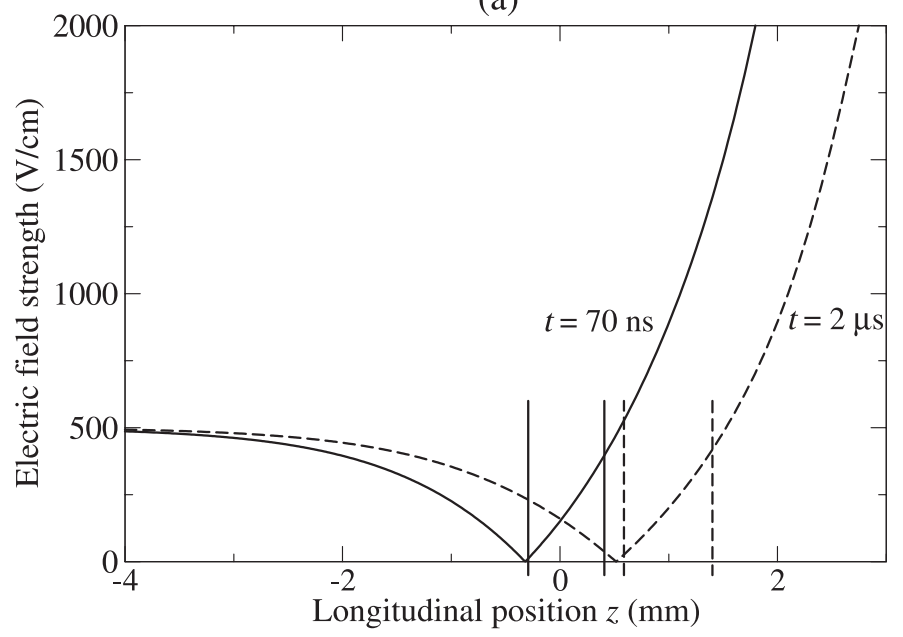

(b)

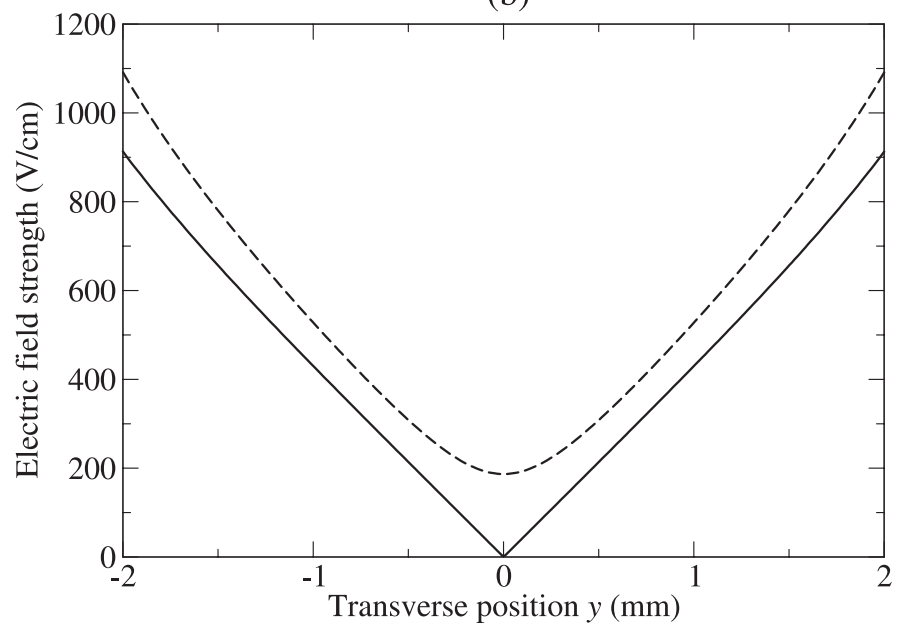

Fig. 2. (a) Electric field strength along the $z$-axis $70 \mathrm{~ns}$ (solid line) and $2 \mu \mathrm{s}$ (dashed line) after photoexcitation calculated for the following set of potentials: $t=70 \mathrm{~ns}: V_{1}=-V_{2}=150 \mathrm{~V}, V_{3}=-V_{4}=-550 \mathrm{~V}$ and $t=2 \mu \mathrm{s}: V_{1}=-V_{2}=150 \mathrm{~V}$, $V_{3}=-V_{4}=-222 \mathrm{~V} . z=0 \mathrm{~mm}$ corresponds to the edge of plates 1 and 2 facing plates 3 and 4 , as shown in Figure $1 \mathrm{a}$. The full and dotted vertical lines limit the regions where the electric field strength lies between $0 \mathrm{~V} / \mathrm{cm}$ and the Inglis-Teller field at $n=22$. On lines parallel to the $z$-axis, the distribution of electric field strengths has a similar shape but the minimum is slightly above $0 \mathrm{~V} / \mathrm{cm}$. (b) Electric field strength along the $y$-axis on the lines $z=-0.4 \mathrm{~mm}$ (solid line) and $z=0$ (dashed line). The potentials that were applied are $V_{1}=-V_{2}=150 \mathrm{~V}$ and $V_{3}=-V_{4}=-550 \mathrm{~V}$.

Table 1. Parameters used in the simulations.

\begin{tabular}{ll}
\hline parameter & value \\
\hline longitudinal velocity & $590 \mathrm{~m} / \mathrm{s}$ \\
longitudinal temperature & $0.6 \mathrm{~K}$ \\
valve orifice diameter & $0.5 \mathrm{~mm}$ \\
skimmer orifice diameter & $0.5 \mathrm{~mm}$ \\
valve-skimmer distance & $12 \mathrm{~cm}$ \\
skimmer-laser beam distance & $7 \mathrm{~cm}$ \\
laser beam-detector distance & $20.5 \mathrm{~cm}$ \\
VUV-laser beam width & $0.3 \mathrm{~mm}$ \\
\hline
\end{tabular}

\section{Results}

\subsection{Experimental results}

Figure 3 shows the TOF spectra measured for the $n=18$, $k=0, n=18, k=-12$ and $n=17, k=11$ states of argon as dotted, solid and dashed lines, respectively. The kinetic energy differences of the $k=-12$ and $k=11$ atoms relative to the $k=0$ atoms are $55 \mathrm{~cm}^{-1}$ and $-51 \mathrm{~cm}^{-1}$, respectively, and are identical to the kinetic energy differences obtained in our earlier experiments [8]. If the small asymmetry in the experimental data is excepted the agreement between simulations and experiment is excellent.

The corresponding images of the Rydberg atom beam on the MCP detector are displayed in the upper half of Figure 4 and the intensity distributions along the $x=0$ and $y=0$ lines are shown in Figure 5, where the $y=0$ line corresponds to the dimension parallel, and the $x=0$ line to the dimension perpendicular to the VUV beam. All three images in the upper half of Figure 4 have the same intensity distribution along the $x$-axis (see also Fig. 5b).

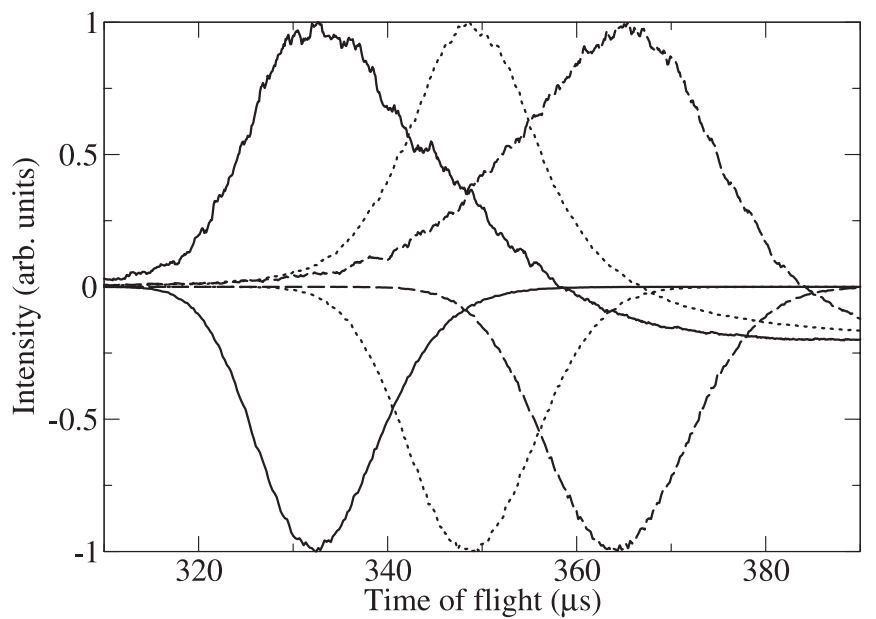

Fig. 3. TOF spectra for $n=18, k=0$ (dotted line), $n=$ $18, k=-12$ (solid line) and $n=17, k=11$ (dashed line). The experimental spectra point upward, the simulated spectra point downward.

This observation is in agreement with the fact that no forces act on the particles in this dimension because of the homogeneous nature of the electric field distribution. The image obtained for the particles in the $k=0$ state (panel (a) in Fig. 4) directly reflects the experimental geometry because these particles do not feel any forces. This measurement enables the characterization of the spatial and velocity distributions of the beam in the absence of any field effects. The image has an elliptical shape with the long axis along the $x$-dimension (Full width at half maximum FWHM $=4.6 \mathrm{~mm}$ ) and the short axis along the $y$-dimension (FWHM $=2.1 \mathrm{~mm}$ ). Assuming 
(a)
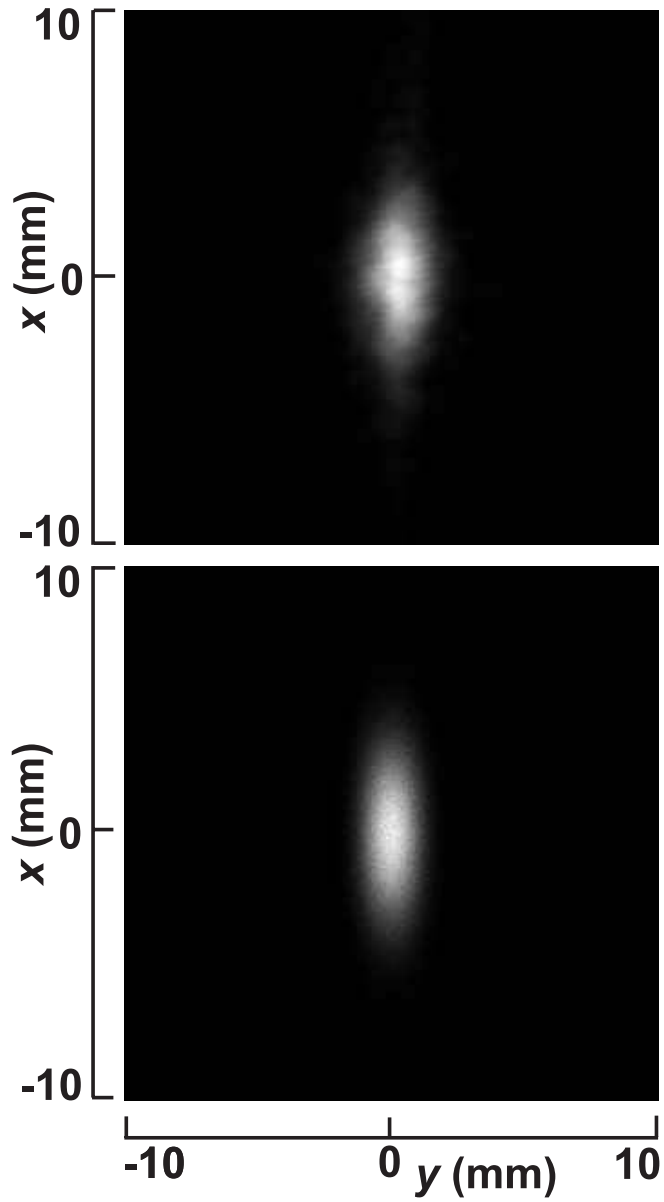

(b)
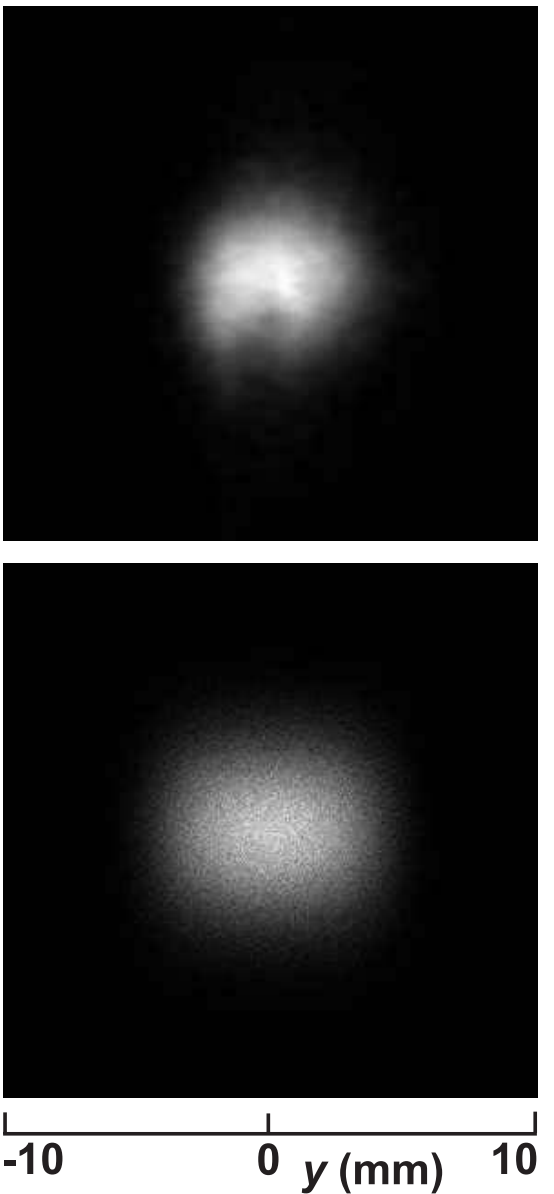

(c)
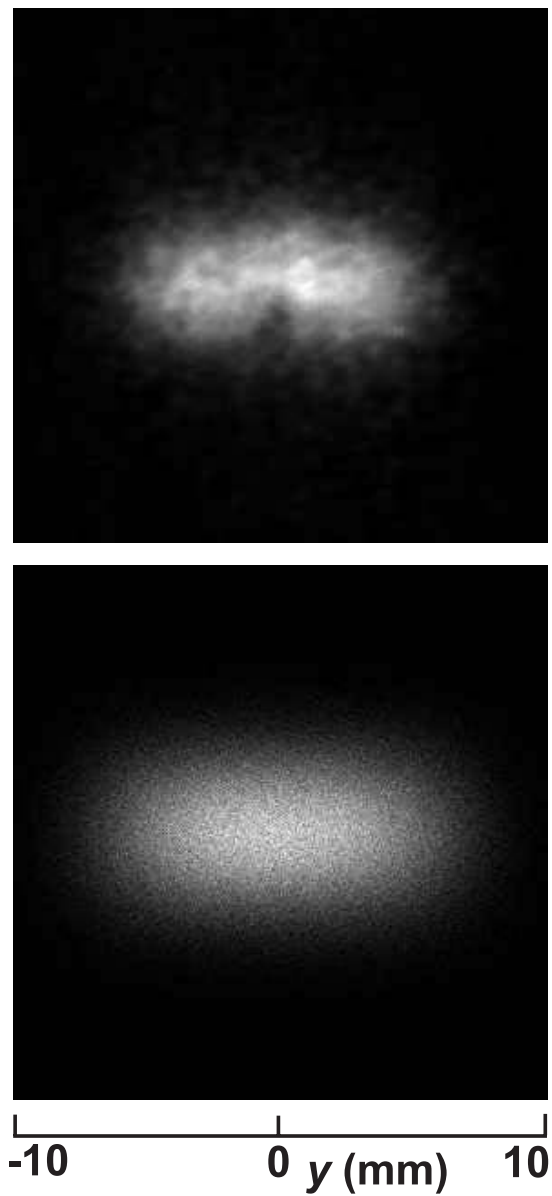

Fig. 4. CCD-camera images of the three excited Stark states. Left: $n=18, k=0$, middle $n=17, k=11$, right $n=18$, $k=-12$. The simulations are shown below the CCD camera images.

(a)

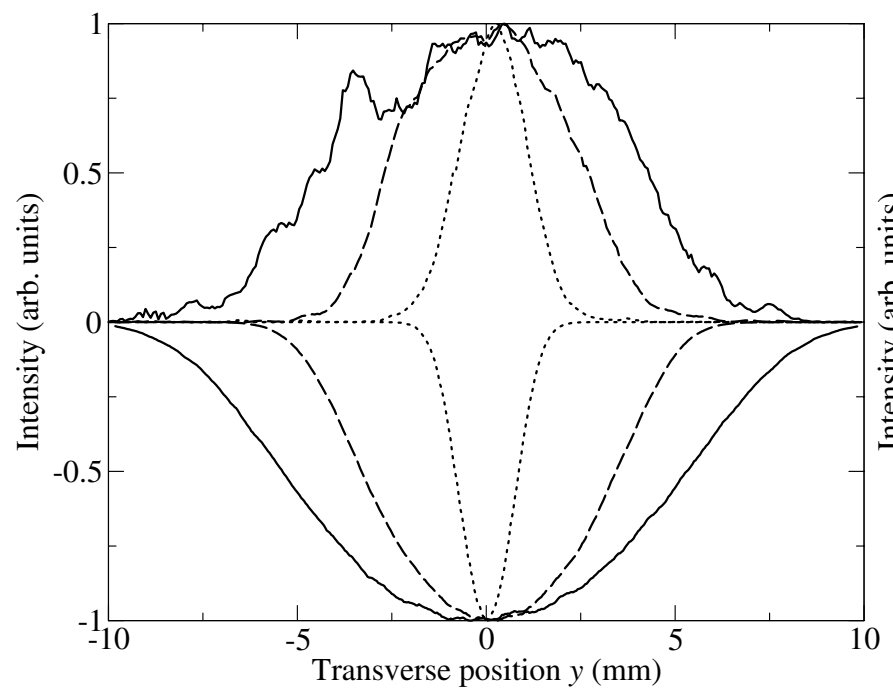

(b)

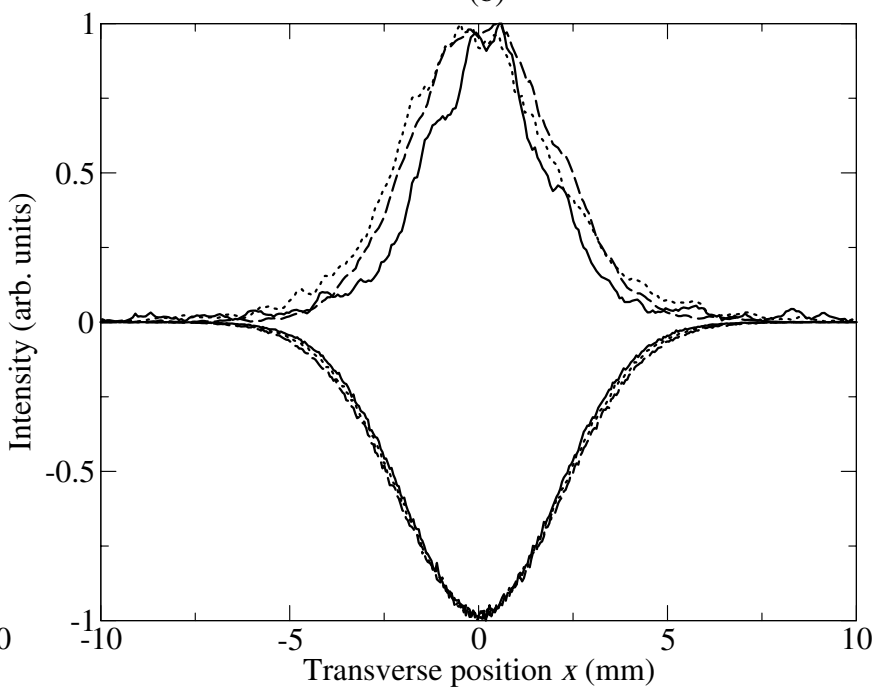

Fig. 5. Line profiles taken from the CCD-camera images (pointing upward) and taken from the simulations (pointing downward) for $n=18, k=0$ (dotted line), $n=18, k=-12$ (solid line) and $n=17, k=11$ (dashed line). (a) Line profiles in the direction perpendicular to the VUV-beam ( $y$-direction). (b) Line profiles in the direction parallel to the VUV-beam ( $x$-direction). 


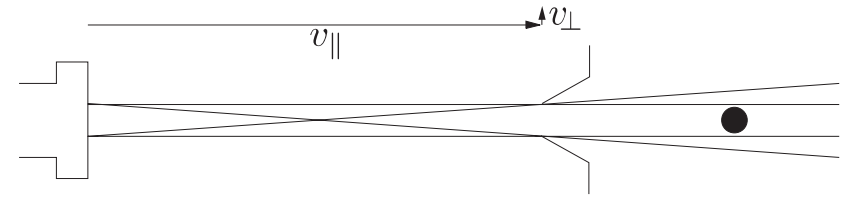

valve

Fig. 6. Valve and skimmer geometry to estimate the maximum transverse velocity in the absence of beam-skimmer interactions. Shown are the trajectories that have maximum transverse velocity and the outer trajectories for which the transverse velocity is zero.

that the effective nozzle opening is equal to the actual nozzle opening and that the skimmer rejects all particles that have too large a transverse velocity, but has no effect on the velocity distribution of the particles that pass through it, the image can be constructed geometrically from the known nozzle and skimmer orifice diameters $(0.5 \mathrm{~mm})$ and the known nozzle-skimmer and skimmerdetector distances (see Tab.1 and Fig. 6). Under these conditions, a circular image of radius $1.7 \mathrm{~mm}$ with a flat intensity distribution is expected. The actual image, however, is significantly broader in the $x$-dimension. Because the VUV beam crosses the supersonic beam along the $x$ axis, it probes the complete spatial and velocity distribution along this axis. From the unexpectedly large width of the distribution along the $x$-axis, one can therefore conclude either that the effective nozzle opening is much larger than the actual nozzle opening or that the skimmer perturbs the particles during their flight through the orifice and broadens the velocity distribution as already observed in previous studies [11]. The broadening of the velocity distribution by the skimmer was observed independently in the same apparatus as a Doppler broadening in a high-resolution spectroscopic measurement of the hyperfine structure of the $(5 p)^{6}{ }^{1} \mathrm{~S}_{0} \rightarrow(5 p)^{5} 7 d[3 / 2](J=1)$ transition of xenon [12]. As will be discussed in Section 4.2, this broadening can be quantitatively reproduced if one assumes that a rethermalization of the beam in the transverse dimensions to a temperature $T_{t}=100 \mathrm{mK}$ takes place at the skimmer. Under these conditions, the waist of the supersonic beam at the point of intersection with the VUV laser beam is predicted to be $1.1 \mathrm{~mm}$ (FWHM).

The reduced size of the image in the $y$-dimension compared to that in the $x$-dimension is a consequence of the fact that the size of the VUV laser beam $(0.3 \mathrm{~mm})$ is smaller than that of the molecular beam and selects the low transverse velocity components in that dimension, leading to a distribution approximately described by a temperature of $15 \mathrm{mK}$.

The images in Figures $4 \mathrm{~b}$ and $4 \mathrm{c}$ for the low-field seeking $k=11$ and the high-field seeking $k=-12$ Rydberg levels can be qualitatively understood by considering the electric field distribution in the deceleration stage. Both images show a broader distribution along the $y$-axis than that corresponding to the $k=0$ Stark state (panel a). In the case of the $k=-12$ state, the inhomogeneity of

the electric field in the $y$-direction leads to an acceleration away from the $z$-axis over the whole deceleration stage and thus to the widest image on the detector. In contrast, the particles excited to the $k=11$ state are first accelerated towards the $z$-axis so that the Rydberg atom beam, which initially diverges away from the $z$-axis, is refocused. After crossing the $z$-axis, the beam diverges again on its way to the detector. The path to the detector after the crossing is long enough that the image size along the $y$-axis is larger than for the $k=0$ particles.

A similar behaviour was observed in measurements made with Rydberg Stark states with $n=15$ to 16,18 to 19 and 21 to 22 . The effects, however, become less pronounced at increasing $n$, primarily as a result of the reduced maximal field strength imposed by the necessity of carrying out the experiments below the Inglis-Teller field.

In the measurements using time-dependent fields, the width of the spatial distribution in the $y$-direction at the detector was always found to be larger than for the $k=0$ atoms. The reasons for this behaviour are that the atoms in $k<0$ Stark states undergo an accelerating force away from the beam propagation axis over the whole length of the deceleration stage and that the atoms in $k>0$ Stark states are subject to such a strong accelerating force toward the beam propagation axis that the particles are brought back to the axis close to the end of the deceleration stage and the beam diverges on its way to the detector.

The focusing of the Rydberg atom beam could be demonstrated directly in experiments with timeindependent fields. In this case, the forces acting on the particles are reduced and the focal point can be brought close enough to the detector that the size of the image in the $y$-dimension is reduced. This behaviour is demonstrated in Figure 7 which compares CCD-camera images recorded for atoms in $n=16, k=0$ (panel (a)) and $n=15 k=9$ (panel (b)) states, respectively, using $V_{1}=-V_{2}=880 \mathrm{~V}$ and $V_{3}=V_{4}=0$. In this voltage configuration, the atoms in $k>0$ states are accelerated toward the $z$-axis. Because the focal point for the beam of $k=9$ atoms now lies close to the detector, the width of the particle distribution in the $y$-dimension is narrower $(\mathrm{FWHM}=0.9 \mathrm{~mm})$ than in the case of the $k=0$ atoms $(\mathrm{FWHM}=1.4 \mathrm{~mm})$. Figure 7 thus directly demonstrates that a Rydberg particle beam can be focused in one dimension using our deceleration stage, as was indirectly implied by the time-dependent measurements described above.

\subsection{Simulations}

The parameters listed in Table 1 and the potentials applied to the four electrodes of the deceleration stage determine the experimental set-up completely and form the basis of the particle trajectory calculations. Because of the elongated shape of the image of the $k=0$ particles discussed in the previous subsection, an additional parameter describing the transverse velocity distribution of the particles leaving the skimmer had to be introduced and 
(a)

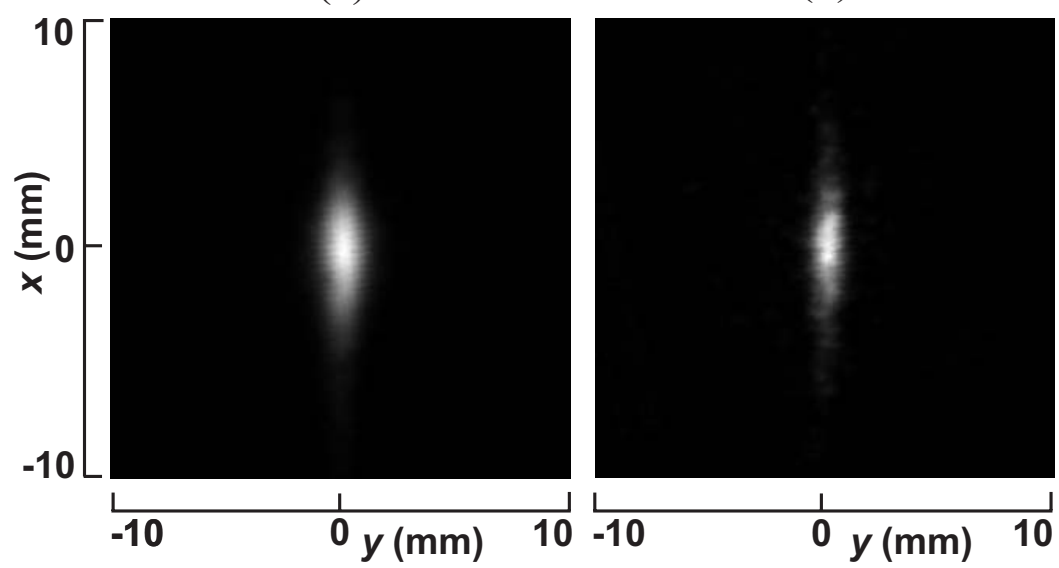

(c)

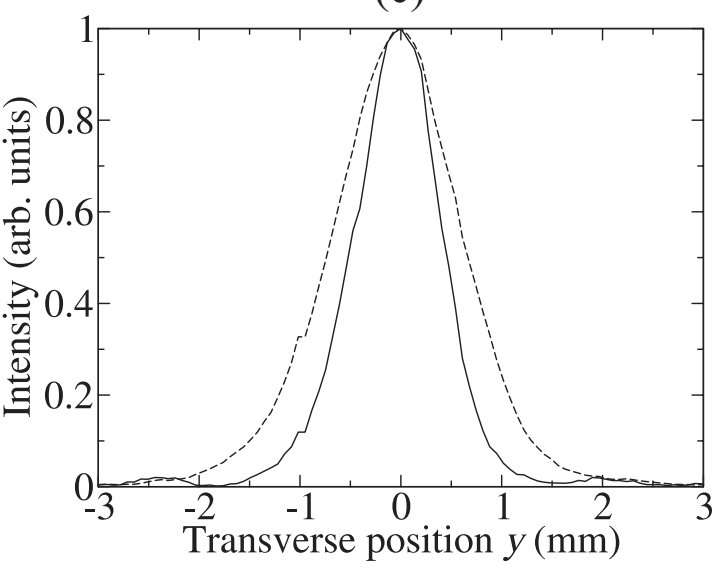

Fig. 7. CCD-camera images of the Rydberg atom cloud in $n=16, k=0$ state (a) and in the $n=15, k=9$ state (b) obtained by operating the deceleration stage with time-independent voltages $V_{1}=-V_{2}=880 \mathrm{~V}$ and $V_{3}=V_{4}=0 \mathrm{~V}$. The dashed (full) line in (c) represents the measured intensity distribution along the $y$-dimension at $x=0$ for the $k=0(k=9)$ state.

optimized to obtain a satisfactory agreement with the experimental results. An optimal value of $100 \mathrm{mK}$ was found and led to the simulated images shown in the lower half of Figure 4 and the calculated TOF and spatial distributions displayed as inverted traces in Figures 3 and 5 . Each simulated image was constructed from 1000000 trajectories sampling the whole space of initial velocities and positions, taking into account the Maxwell-Boltzmann velocity distributions and assuming a Gaussian profile with a width of $0.3 \mathrm{~mm}$ for the laser beam. The good agreement between the experimental and the simulated images obtained using a single adjustable parameter clearly indicates that the deceleration stage is well characterized.

The simulations can be used to highlight features not directly accessible from the raw experimental data. Firstly, the simulations of the trajectories of the high-field seeking Rydberg particles show that these particles are brought back to the $x z$-plane in a narrow range of positions approximately $1 \mathrm{~cm}$ after leaving the deceleration stage. The analysis of the trajectories together with the results displayed in Figure 7 make us confident that the electrode set-up could be easily modified to achieve a perfect focus of the Rydberg particles. Secondly, the simulations enable the determination of the velocity distributions in all three dimensions and of the changes in these distributions occurring during the deceleration/acceleration process. Effective translational temperatures can then be estimated by fitting Maxwell-Boltzmann distributions to these velocity distributions. The initial effective temperature along the $x$-axis amounts to $100 \mathrm{mK}$ and remains unaffected during the acceleration/deceleration. The initial effective temperature along the $y$-axis is only $\sim 15 \mathrm{mK}$ immediately after the Rydberg states have been generated by the VUV laser pulse. This temperature does not change for the $k=0$ states but increases to $300 \mathrm{mK}$ and $700 \mathrm{mK}$ for the $k=11$ and $k=-12$ Stark states, respectively.

Because of the refocusing effect of the deceleration stage, the translational temperature along the $y$ dimension will always be smaller for the decelerated par- ticles than for the accelerated particles. Adjustments of the electrode separation and voltages can lead to a significant reduction of this temperature, however, at the cost of the effectiveness of the overall deceleration as illustrated by the time-independent deceleration measurements displayed in Figure 7.

\section{Conclusion}

The properties of our Rydberg Stark decelerator have been characterized in a set of time-of-flight and imaging measurements of trajectories of argon atoms excited to a variety of Stark states in the range of principal quantum numbers $n=15$ to 25 . The characterization included the determination of the size of, and the velocity distribution in the supersonic atomic beam after passing through the skimmer, of the size of the VUV beam and, most importantly, of the full three-dimensional velocity distribution of the Rydberg particles before and after the deceleration.

Under optimal deceleration conditions for high-fieldseeking Stark states using time-dependent fields, we find that, although the transverse velocity distribution is broadened and the mean kinetic energy in the transverse dimensions is increased, this increase only amounts to $10^{-23} \mathrm{~J}\left(0.5 \mathrm{~cm}^{-1}\right)$ and thus represents an almost insignificant fraction of the decrease in kinetic energy of $1.0 \times 10^{-21} \mathrm{~J}\left(50 \mathrm{~cm}^{-1}\right)$ in the longitudinal direction. The temperatures in the transverse dimensions of $100 \mathrm{mK}(x)$ and $300 \mathrm{mK}(y)$ for these particles are sufficiently low that trapping can be envisaged.

We thank René Gunziger, M. Vecellio, H. Schmutz and M. Andrist for their help in the construction of the decelerator and Prof. T.P. Softley (Oxford) and Dr. S. Procter (Oxford) for useful discussions. This work is supported by ETH Zurich under Project TH-28/02-3 and the Swiss National Science Foundation. 


\section{References}

1. J. Doyle, B. Friedrich, R.V. Krems, F. Masnou-Seeuws, Eur. Phys. J. D 31, 149 (2004)

2. H.L. Bethlem, G. Meijer, Int. Rev. Phys. Chem. 22, 73 (2003)

3. J. van Veldhoven et al., Eur. Phys. J. D 31, 337 (2004)

4. S.A. Schulz et al., Phys. Rev. Lett. 93, 020406 (2004)

5. E. Vliegen, F. Merkt, J. Phys. B: At. Mol. Opt. Phys. 38, $1623(2005)$

6. T.P. Softley, Int. Rev. Phys. Chem. 23, 1 (2004)

7. S.R. Procter, Y. Yamakita, F. Merkt, T.P. Softley, Chem. Phys. Lett. 374, 667 (2003)
8. E. Vliegen, H.J. Wörner, T.P. Softley, F. Merkt, Phys. Rev. Lett. 92, 033005 (2004)

9. T.F. Gallagher, Rydberg Atoms (Cambridge University Press, Cambridge, 1994)

10. U. Hollenstein, H. Palm, F. Merkt, Rev. Sci. Instr. 71, 4023 (2000)

11. Atomic and Molecular Beam Methods, edited by G. Scoles (Oxford University Press, Oxford, 1988)

12. Th.A. Paul, F. Merkt, J. Phys. B: At. Mol. Opt. Phys. 38, $4145(2005)$ 